\title{
Design of Simulation Experiment Platform for C2 System Agility
} RAO Jiaren ${ }^{1, a}$, LIN Xin ${ }^{1}, \mathrm{CHU}$ Wei ${ }^{1}$

${ }^{1}$ Science and technology on information systems engineering laboratory, Nanjing 210007 , China araojiaren19871210@163.com

Key words: Command and control system, Agility, Simulation Experiment Platform

\begin{abstract}
Researched on conceptual model of C2 system agility, and designed simulation experiment platform. By using Agent, the simulation resources were packaged to be cooperative, to meet needs of agility. The agile C2 System was built according to military mission, it can sense changes and evolve, which makes command and control system has the ability of collaborative structural adjustment. Finally, battlefield scenario and evaluation index of C2 system agility were given based on agility motivation.
\end{abstract}

\section{Introduction}

2014, the Department of Defense Command and Control Research Program (CCRP) hold the 19th International Command and Control Research and Technology Symposium (ICCRTS) with the theme "C2 Agility: Lessons Learned from Research and Operations", aims to assess progress Agility command and control "implementation" in the process achieved, and in order to continue the process from theory to push practice what needs to be done to do. Alberts said that agility was the capability to successfully effect, cope with, and/or exploit changes in circumstances, can be directly observed only when this capability has been manifested, "How can we measure a system's Agility IQ?" and "What is the requisite amount of agility that is required?" were discussed in his paper[1].In document [2], Yong-Gang Qin said that there are two logics for the technological system (TS) and organizational system (OS) to interact with each other which affect the C2 agility, One is TOT logic that means C2 information flow from technological system nodes to organizational system, and then back to TS nodes, the other is OTO logic that means C2 information flow from OS nodes to TS nodes, and then back to OS nodes. The key is to develop common, standards, measurable, mining, visualization command information both for command personnel, but also for computer processing. Chan et al [3] established a mathematical model to describe the expertise and developed, and based on this model, developed an simulation agent which can simulate artificial agent under different conditions with good performance.

Domestic scholars have done some research on agility theory, too. Mainly of them focused on the study of agile C2 organization. In 2009, Lu Zhenya[4] followed the developing trend of C2 organization effectiveness measurement research, introduced exploratory analysis into the process of measure of $\mathrm{C} 2$ organizational effectiveness, mainly investigated the exploratory analysis method used in measure of $\mathrm{C} 2$ organizational effectiveness, constructed an exploratory analysis framework for measure of $\mathrm{C} 2$ organizational effectiveness, and explored the related technology and methods of importance. In 2010, document [5] proposed the model of agile C2 organization, an approach of agile of $\mathrm{C} 2$ organizational structural design under the certain task environment, as well as an approach of agile $\mathrm{C} 2$ organizational structural adjust under the uncertain task environment.

From the research home and abroad, research of allegations agility is still focused on C2 organization. However, as a mean and direct tool, command and control system agility lack attention, in view of this, this paper focus on the concept of agile command and control system model, and simulation platform design with the theoretical guidance, to provide the basis agility simulation. 


\section{Agility conceptual model of command and control system}

Agile command and control system refers Information Grid environment, it is possible to diversify the combat mission driven, fast, dynamic organization wide online distribution grid, belong to different organizations, different characteristics of various system resources and property at the right time collaboration on the appropriate node function properly formed to meet the needs of the mission command and control system. Through using of flexible and efficient and organizational methods of system resources to successfully meet unexpected, complex battlefield environment.

Agility is the ability to successfully cope with change in circumstances [6]. Thus, Agility explicitly requires change - the ability to deal with a dynamic situation. Change is a fundamental prerequisite for agility, as well as motivation of agility. No changes, command and control system does not need to be agile but also to maintain a stable output capability. Changes typically include changes in the external environment and internal state change of command and control system, and the new constantly changing interplay between these two changes produced. Changes in the external environment, including system intelligence-ensured interrupted, command post damaged, and so on; internal state changes of the system include system resource damaged, task overload, adding new resources, and so on.

Since agility refers ability to successfully respond to various situations change, agile command and control system must maintain an acceptable level of effectiveness and efficiency, through agility recovery system performance, and keep stable output of system performance. The conceptual model shown in Figure 1:

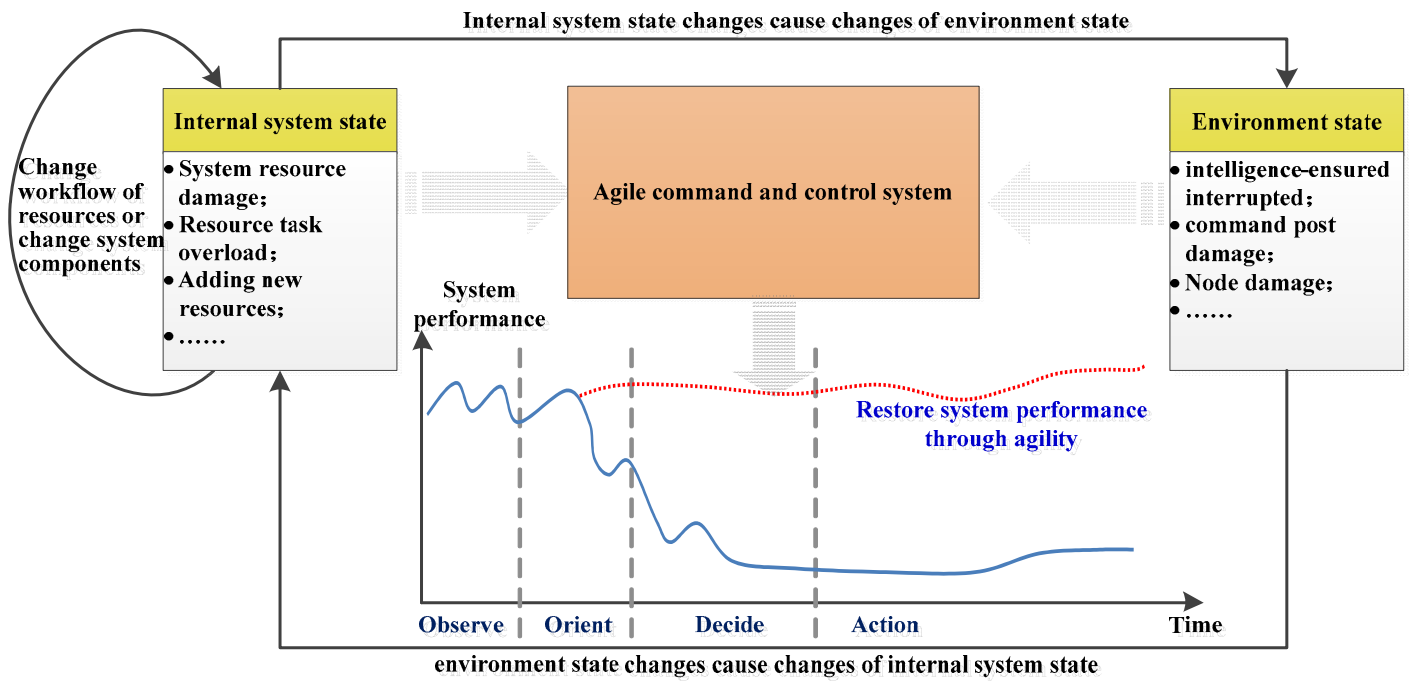

Fig1. Schematic agility conceptual model of command and control system

\section{Simulation Experiment Platform Design}

Command and control system cannot exist in isolation, only in the operations process can play its effectiveness, therefore, in-depth study of command and control system agility is also dependent on building simulation experiment platform. This article will focus on the design of simulation-based experiment platform, lay the foundation for the command and control system agility test.

Simulation platform includes various types of simulation resources, Operational support environment, experiment management system, database system, and evaluation system. Among them, the simulation resources including intelligence processing subsystem, strategic warning subsystem, command posts subsystem and weapon against subsystem, each subsystem consists of several simulators (including radar, various command posts and weapons, and so on), the simulators are regarded as resources deployed in grid network. Operational support environment includes a 
number of simulation Agent and a master. Experiment Management System consists of scenario editor, environment configuration, scenario generation, and experiment direction and simulation control. Database system includes resource database, configuration database, archival repository, and performance database. Evaluation system consists of data collection and the display of simulation results. The agility simulation platform shows in Figure 2.

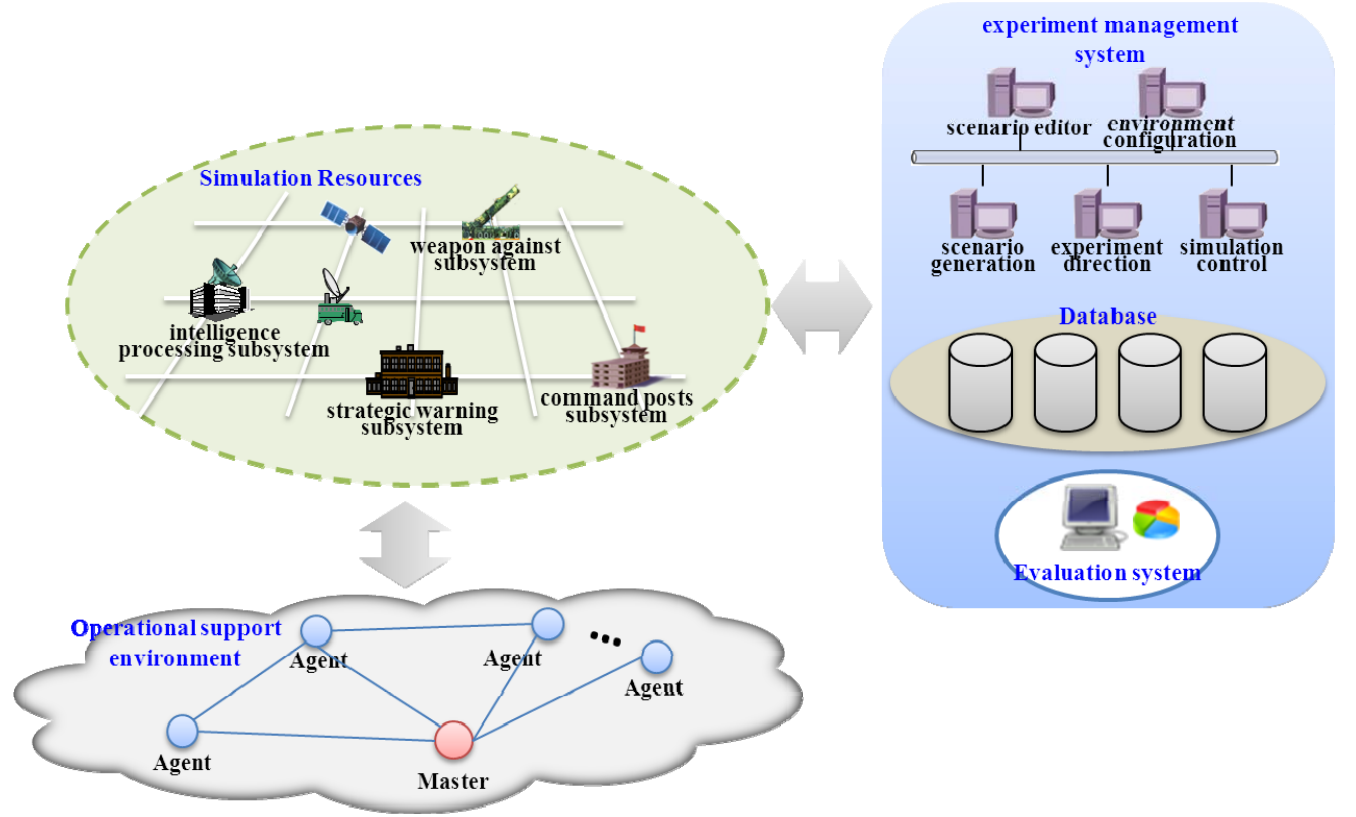

Fig2. Schematic simulation platform

In order to improve the ability to respond flexibly, the Construction and operation of agile command and control system focuses on how to organize system resources with dynamic collaboration, the core feature is task-driven and collaborative operational restructuring. Our C2 system based on the simulation experiment platform is also driven by mission, agile command and control system built based on different tasks [7], and when internal or external state changes, the dynamic evolution been aroused to adjust its structure to maintain system performance.

Agent-based simulation systems running structure shown in Figure 3, For a given task, the master parses the resource workflow file, and send the rules to each resource Agent, then each Agent is bound to a resource, interaction of resources and perception of changes are achieved though these Agents.

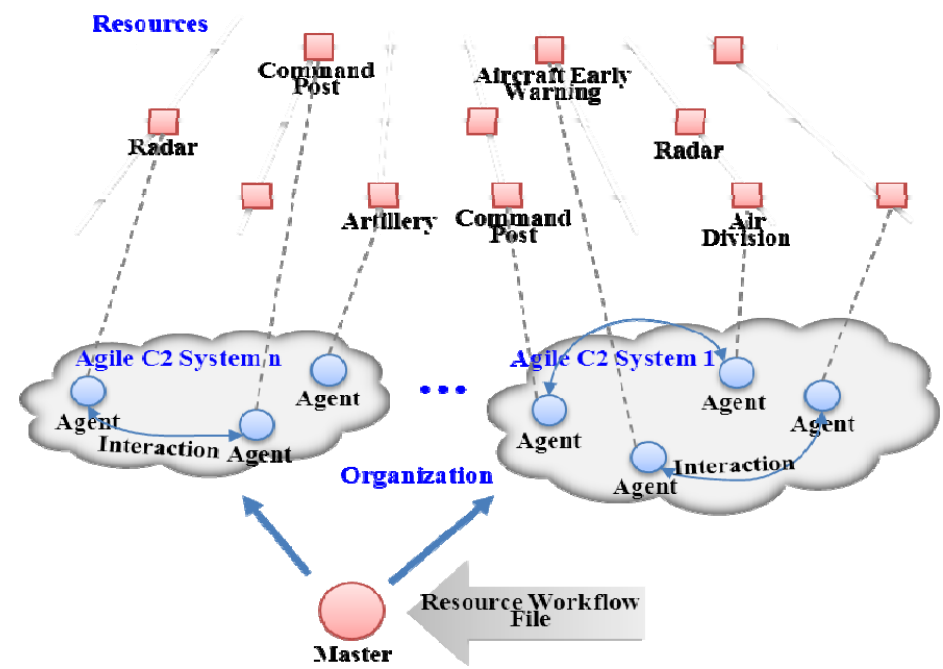

Fig3. System operation Chart

Dynamic battlefield environment change is inevitable during the running of command and control system, furthermore, system structural adjustment and internal node failures are still possible. To ensure the system's agility, the evolution process exists in the system life. This 
evolution is similar to the OODA (Observe, Orient, Decide, Action) loop, which is an autonomous optimization cycle.

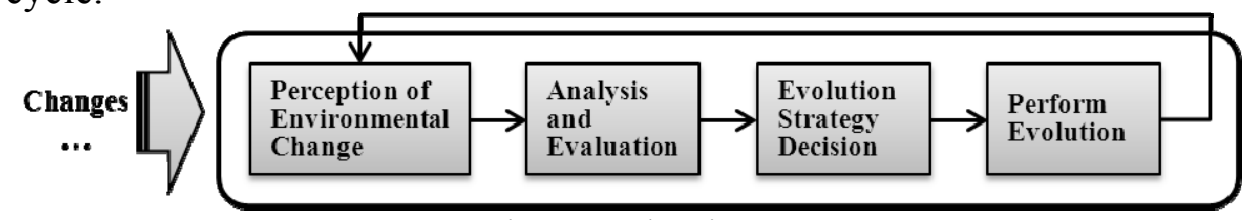

Fig.4 Evaluation

As fig.4 shows, the system evolution process includes perception of environmental change, analysis and evaluation, the evolution strategy decision, and performs evolution. Among them, the perception of environmental change stage is perceiving external and internal state changes by resource, and providing notification mechanisms of changes.

In analysis and evaluation stage, considering the specific evolution strategy, policy constraints and generation rules, system tasks quantitative analysis and evolution are achieved after change event notification received.

In the evolution strategy decision stage, the mechanism of evolution, structural adjustment and restructuring are oriented and decided. Finally, when stepping to put evolution strategy into practice, C2 system makes response to changes and adjusts structure itself. Evolution often requires continuous feedback to adjust continuously optimized to meet the tasks and change needs.

\section{experiment design}

Considering the typical cases of internal and external states changes, intelligence-ensured interrupted, command post damage, and resource task overload, designs following scenario.

Intelligence-ensured interrupted:

Several radars of blue team is attacked and damaged by red team, the regional intelligence displayed in command post COP (common of picture) seams abnormal, the staff on duty immediately issued a request for information security.

The command and control system executes analysis and evaluation after receiving instruction. Searches for functionally equivalent resources for perception in grid network, and add them to the running $\mathrm{C} 2$ system, the regional intelligence displayed in COP turns to be normal. At the same time, we can judge the agility by focusing on the amount of system intelligence and resource.

Command post damage:

A command post of red team is destroyed by bombers of blue team, analysis and evaluation process is aroused after Agent/resource perceived this situation. System searches the candidate set of resources for finding out a command post resource to replace the destroyed one, and adds the resources to the command and control system, while makes the resources collaborative to restore the system capabilities. At this point, time-consuming, amount of system resources and real information flow in COP can be used to determine the agility of $\mathrm{C} 2$ system.

Resources task overload:

Several planes of blue team break into the guard area of A air division, A air division is asked to intercept the planes in its guard area, but, the capacity is not sufficient to intercept all of the aircrafts. Therefore, system start evolution process, after analysis and evaluation, the task balancing mechanism is aroused to help accomplishing the task, and B air division nearby is assigned to intercept the last aircrafts. There, the assignments of each air division and time-consuming reflects the system agility.

\section{Summary}

Embark from agility motivation, analyzed the required ability and desired effect of command and control system to maintain stable output, reasoning summarized agility conceptual model of command and control system.

Based on the study of agility, and designed simulation platform. Built agile $\mathrm{C} 2$ system based on combat missions, the simulation resources were packaged to be cooperative, when the internal or 
external state changes, $\mathrm{C} 2$ system calls evolution process, collaborative adjusts resource structure to restore system performance or to complete the assigned task.

Furthermore, considering the typical cases of internal and external states changes, intelligence-ensured interrupted, command post damage, and resource task overload, designed a scenario. evaluation indexes for all types of situations were given, which makes solid foundation for verifying $\mathrm{C} 2$ system agility.

\section{References}

[1] David S. Alberts. Agility Quotient. 19 ${ }^{\text {th }}$ ICCRTS.2014.

[2] Yong-Gang Qin, Hong Chen, Xiang-Li Xu. Essential Structures of C2 Subsystems and Interaction Logics to Agility. $19^{\text {th }}$ ICCRTS.2014.

[3] Kevin Chan, Jin-Hee Cho, etc. Providing Agility in C2 Environments Through Networked Information Processing: A Model of Expertise. 19 ${ }^{\text {th }}$ ICCRTS.2014.

[4] Liu Zhenya. A Research of Exploratory Analysis for Measure of C2 Organizational Effectiveness. Graduate School of National University of Defense Technology. 2009.

[5] Haixiao Liu. Design and Adjust Methodology of Command and Control Organization. Graduate School of National University of Defense Technology Changsha, 2010.

[6] D.S. Alberts, Agility Advantage, Washington[M], DC: CCRP Publications Series, 2011.

[7] Yan Jingjing, Duanmu Zhuyun, Zhou Xiaoming, etc. Research of the Dynamic Reconfiguration Mechanism for C2 Communities of Interest[D]. Command Information System \& Technology.2010,1(4). 